\title{
Minimal inductance for axisymmetric transmission lines with radially dependent anode-cathode gap
}

\author{
Eduardo M. Waisman and M. E. Cuneo \\ Sandia National Laboratories, P.O. Box 5800, Albuquerque, New Mexico 87185-1193, USA
}

(Received 25 July 2009; published 30 September 2009)

\begin{abstract}
We extend the variational calculus technique for inductance minimization of constant gap axisymmetric transmission lines (TL), introduced by Hurricane [J. Appl. Phys. 95, 4503 (2004)], to the case in which the anode-cathode gap is a linear function of the midgap radius. The full analytic optimal midgap solution curve $z(r)$ yielding minimum inductance is obtained in terms of a single parameter $\rho_{0}$, determined numerically by imposing that $z(r)$ goes through prescribed end points. The radius of curvature $\rho(r)$ of the optimal curve is obtained everywhere the function is defined, even outside of the end point range, and it is shown that a convenient choice is $\rho_{0}=\rho(0)$. The value of the transmission line inductance is calculated by $1 \mathrm{D}$ numerical quadrature. A simple numerical technique is introduced for TL with nonlinear radial gap dependence.
\end{abstract}

DOI: 10.1103/PhysRevSTAB.12.090401

PACS numbers: 41.20.- $-\mathrm{q}, 52.58 . \mathrm{Lq}$

\section{INTRODUCTION}

Electrically driven pulsed-power generators, such as Sandia's Z [1], utilize transmission lines (TL) to carry the electrical pulse from the power source, generally a capacitor bank, to the load. These TL consist of metallic electrodes separated by a medium such as oil, water, air, vacuum, etc.

In most applications of pulsed power it is desirable to minimize the inductance of the TL to shorten the current rise time and maximize its amplitude. For as long as pulsed power has been used, its practitioners have designed TL trying to minimize inductance. Simultaneously, the TL design must assure adequate electrical insulation during operation to prevent electrical current from shunting across the anode-cathode (AK) TL gap [2,3]. This involves constraints on the AK gap. However, no formal procedure to optimize TL by minimizing inductance given explicit constraints on the AK gap was available, to the best of our knowledge, before the work of Hurricane [4]. In this reference the author solves for the optimal TL of the constant anode-cathode (AK) gap filled with a medium of constant magnetic permeability $\mu_{0}$. We note that the minimization of electron flow current in magnetically insulated transmission lines (MITL) is a common design criterion for multi-mega-ampere, multimodule pulsed-power drivers $[1,2,5]$, and has been done, both implicitly through large numbers of simulations [1], and recently in a more explicit form [5].

Since our research extends the work of Ref. [4], it is useful to briefly review here its approach and results. The inductance for the axisymmetric TL is given by

$$
\begin{aligned}
L & =\frac{\mu_{0}}{2 \pi} \iint_{A} d R d Z / R \cong \frac{\mu_{0}}{2 \pi} \int_{r_{1}}^{r_{2}} w d s / r \\
& =\frac{\mu_{0}}{2 \pi} \int_{r_{1}}^{r_{2}} w \sqrt{1+\left(z^{\prime}\right)^{2}} d r / r .
\end{aligned}
$$

In Eq. (1) $A$ stands for the $(R, Z)$ surface enclosed by the anode and cathode electrode curves in the $R, Z$ plane, obtained by going a perpendicular distance of half the AK gap, $\pm w / 2$ at each point of $z(r)$, the midgap curve. We use lower case $r, z$ to denote points on $z(r)$ and denote the midgap inner and outer points by $\left(r_{1}, z_{1}\right),\left(r_{2}, z_{2}\right), r_{2} \geq$ $r_{1}, z_{2}>z_{1}$, respectively. The TL inductance is invariant with respect to translations along the axial coordinate. We are then free to select $z_{1}=0$, and $z_{2}=l_{z}$ the axial length of the TL.

In Eq. (1) the step involved in going from the surface integral to a 1D one along the midgap curve consists in the approximation,

$$
\iint_{d A} d R d Z / R \cong w d s / r,
$$

where $d A$ is the differential $(R, Z)$ area enclosed by the electrodes and the midgap arclength differential $d s=$ $d r \sqrt{1+\left(z^{\prime}\right)^{2}}, z^{\prime} \equiv d z / d r$. This approximation is valid for small gaps, i.e., $r_{a}-r=r-r_{c} \ll r$, where $r_{a}, r_{c}$ are the anode and cathode radii, respectively. We will discuss this small gap condition again in Sec. II. The geometry is depicted in Fig. 1.

This optimal $z(r)$ that minimizes the inductance $L$ satisfies the Euler-Lagrange equation: 


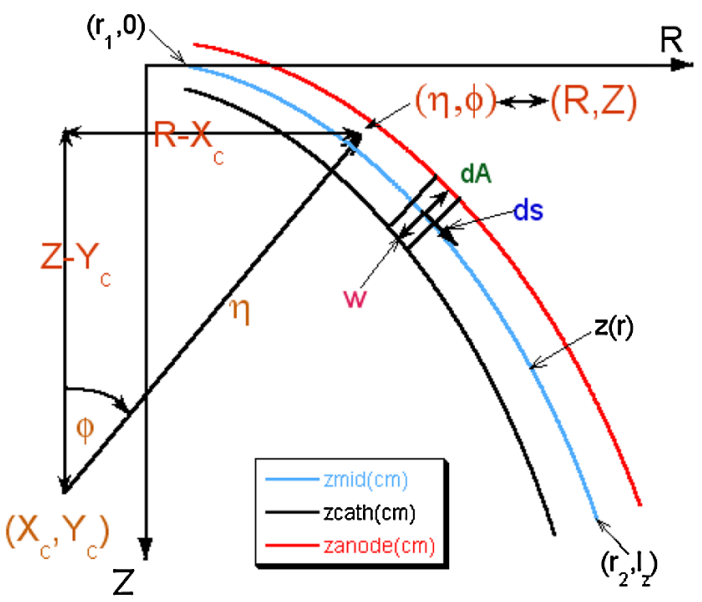

FIG. 1. (Color) Midgap and electrode curves in $(r, z)$ and $(\eta, \phi)$ coordinate systems, geometry and coordinate systems corresponding to an axisymmetric TL. The midgap and electrode curves in $R, Z$ are schematically depicted (cathode in black, anode in red, midgap in blue). The actual TL surface is a figure of revolution around the axis of symmetry. The TL gap $w$ and the differential of arclength $d s$ along the midgap curve are shown, as well as its end points $\left(r_{1}, 0\right),\left(r_{2}, l_{z}\right)$. The polar coordinate system $\eta, \phi$ with variable center of curvature at $\left(X_{c}, Y_{c}\right)$ and $\eta$ in the direction of the local radius of curvature is exhibited. Also indicated is the differential area $d A$ used in the approximation explained in Eq. (2). For definition of terms see text.

$$
\begin{aligned}
& \frac{d}{d r}\left(\partial F / \partial z^{\prime}\right)-\partial F / \partial z=0, \\
& \text { where } F=w \sqrt{1+\left(z^{\prime}\right)^{2}} / r .
\end{aligned}
$$

For $F$ not an explicit function of $z$, it follows from Eq. (3) that $\partial F / \partial z^{\prime}=(w / r) z^{\prime} / \sqrt{1+\left(z^{\prime}\right)^{2}}=$ const, and thus for constant gap, as considered in Ref. [4], we have

$$
z^{\prime} / \sqrt{1+\left(z^{\prime}\right)^{2}}=\kappa r
$$

where $\kappa$ is a constant of integration to be determined by the condition that the midgap curve goes through the prescribed end points. The solution of Eq. (4) is given by a circumference of radius $\rho=1 / \kappa$. It is readily seen that the center of the circumference is given by $X_{c}=0, Y_{c}=$ $\left(r_{2}^{2}-r_{1}^{2}\right) /\left(2 l_{z}\right)+l_{z} / 2$.

A noteworthy feature of this solution is, as already pointed out in Ref. [4], the fact that for $l_{z} \geq \sqrt{r_{2}^{2}-r_{1}^{2}}$ the circumference goes though $r_{2}$ twice, reaching radii greater than $r_{2}$. The value of the maximum radius attained by the midgap circumference is given by $r_{\max }=\rho=$ $\sqrt{\left(Y_{c}-l_{z}\right)^{2}+r_{2}^{2}}>r_{2}$. Therefore $z(r)$ is double valued with $z^{\prime} \rightarrow \pm \infty, r \rightarrow r_{\max }$. This can be explained noticing that minimizing the integral of the function $F\left(z^{\prime}, r\right)$, that is the inductance functional given in Eq. (1), consists of trading off arclength and radius, trying to make the former small and the latter large, along the midgap curve "tied" at its end points. As an example we show in Fig. 2 the electrode and midgap curves optimal solution for $r_{1}=$ $r_{2}=1.5 \mathrm{~cm}, l_{z}=8 \mathrm{~cm}$, and $w=0.8 \mathrm{~cm}$. The vacuum inductance of this optimal TL is $5.5 \mathrm{nH}$, while for the straight coaxial line for the same midgap end points and gap it is $8.7 \mathrm{nH}$.

In some of the applications of axisymmetric TL, for instance vacuum MITL, the AK gap needs to be larger at larger radii. This is to make the ratio of electric and magnetic field $E / B \simeq(V / w) /(I / r)$ a weak function of radius along the TL, here $V$ is the voltage and $I$ is the current at a given point of the TL. In order to be able to design TL taking into account this constraint we generalize the solution of Ref. [4] allowing the AK gap to be an increasing linear function of the radial coordinate:

$$
w(r)=w_{1}+\left(w_{2}-w_{1}\right)\left(r-r_{1}\right) /\left(r_{2}-r_{1}\right)=w_{0}+k r,
$$

where $w_{1}, w_{2}$ are the values of the AK gap at the inner and outer point, respectively, while $k=\left(w_{2}-w_{1}\right) /\left(r_{2}-r_{1}\right)$, and $w_{0}=w_{1}-k r_{1}$ is the extrapolated gap at the axis. We require that $w_{2} \geq w_{1}$, while $w_{0}$ can be either positive or

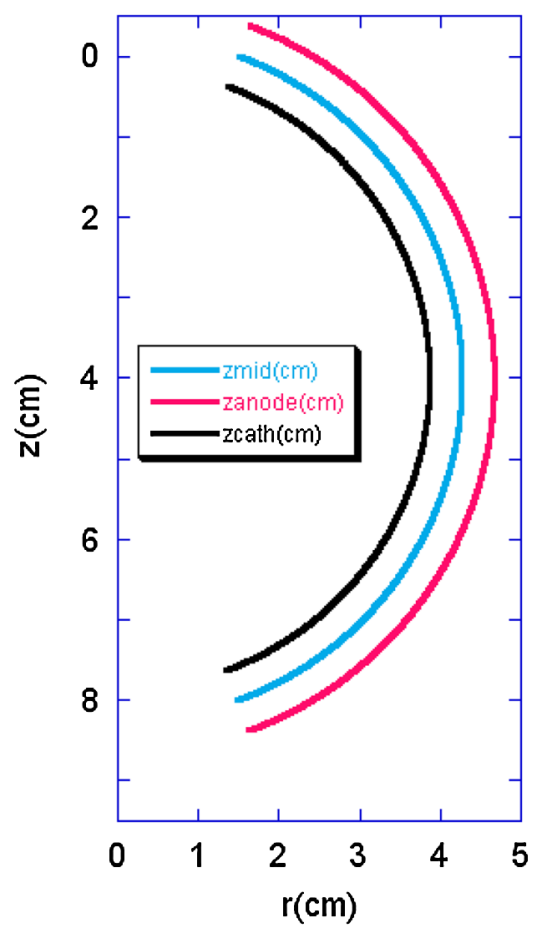

FIG. 2. (Color) Optimal TL for constant gap and equal inner and outer radii. Electrode and midgap curves (cathode in black, anode in red, midgap in blue) for the optimal TL for constant $0.8 \mathrm{~cm}$ gap. The end points of the midgap curve are $r_{1}=r_{2}=$ $1.5 \mathrm{~cm}, l_{z}=8 \mathrm{~cm}$. Notice that this minimum inductance line is given by a circumference in the $R, Z$ plane. In fact, for this example its vacuum inductance is $5.5 \mathrm{nH}$ instead of the $8.7 \mathrm{nH}$ of the straight coaxial line going through the same midgap end points. 
negative. In the latter case, we will prevent solutions that allow the optimal curve reaching radii smaller than $r_{1}$. We add this extra constraint because, as we will see in Sec. II, for $w_{0}<0$ it is possible for the optimal solution to be double valued going through these smaller values of the radial coordinate. Moreover, it is even possible for a certain combination of the boundary conditions, for it to go through unphysical values of negative gap. Thus, we will find that there are certain boundary conditions for negative $w_{0}$ such that no solution exists when this extra constraint $r \geq r_{1}$ for the midgap trajectory is added.

In Sec. II we present the solution, which always exists for positive $w_{0}$, while for the negative case we impose the extra constraint introduced in the previous paragraph. In the same section we also present a technique calculating the inductance for the optimal TL consisting of a straightforward 1D quadrature. We obtain the optimal curve analytically, up to a single dimensionless parameter $\alpha \equiv \rho_{0} k / w_{0}$, which we determine numerically by imposing the condition that the optimal curve attains the prescribed TL end points. Both $k$ and $w_{0}$ are input quantities, thus finding $\alpha$ is equivalent to finding $\rho_{0}$.

In Sec. III we will sketch how to solve for more general dependence of the gap as a function of radius by straightforward numerical integration of the single ordinary differential equation describing the optimal curve. We also give an illustrative example in which the gap varies as the square root of the radius.

In Sec. IV we discuss our results and their limits of applicability.

Before leaving this Introduction it is worth noticing the special case $l_{z}=0$ : TL with constant $z$, i.e. $z^{\prime}=0$ midgap curve. In this case there is no optimization involved and the inductance is simply obtained from Eq. (1); $L=\frac{\mu_{0}}{2 \pi} \times$ $\int_{r_{1}}^{r_{2}} w d r / r$. The observation in Ref. [4] that for TL with constant $z$ midgap curve, the minimum arclength coincides with the minimum inductance is also valid for variable gap.

\section{OPTIMAL SOLUTION FOR LINEARLY INCREASING IN RADIUS AK GAP}

For the gap dependence of Eq. (5) we obtain from Eq. (3) the corresponding differential equation to that of Eq. (4):

$$
z^{\prime} / \sqrt{1+\left(z^{\prime}\right)^{2}}=C r / w
$$

where $C$ is a constant of integration. We rewrite Eq. (6) as an ordinary differential equation in dimensionless form:

$$
y^{\prime}=x / \sqrt{(1+\alpha x)^{2}-x^{2}}, \quad \alpha \equiv k \rho_{0} / w_{0},
$$

where $x \equiv r / \rho_{0}, y \equiv z / \rho_{0}$, and $y^{\prime} \equiv d y / d x=z^{\prime}$. To obtain the dimensionless Eq. (7) we have introduced the scale length parameter $\rho_{0}$, defining it as $\rho_{0}=w_{0} / C$. Thus Eq. (7) contains the single free dimensionless parameter $\alpha$. We will show below that $\rho_{0}$ is the radius of curvature of $z(r)$ at $r=0$.

The radius of curvature for $z(r)$ is given by [6]

$$
\rho(r)=\left[1+\left(z^{\prime}\right)^{2}\right]^{3 / 2} / z^{\prime \prime}=\rho_{0}\left[1+\left(y^{\prime}\right)^{2}\right]^{3 / 2} / y^{\prime \prime},
$$

where $z^{\prime \prime} \equiv d^{2} z / d r^{2}=y^{\prime \prime} / \rho_{0}$ and $y^{\prime \prime} \equiv d^{2} y / d x^{2}$.

From Eq. (7) we obtain $y^{\prime \prime}$ and find

$$
\rho=\rho_{0}(1+\alpha x)^{2} .
$$

From Eq. (9) it is then apparent that at $x=0, \rho(0)=\rho_{0}$, which substantiates the statement we made in the abstract of this article on the meaning of $\rho_{0}$.

Likewise we can find the center of curvature for the optimal curve [6]:

$$
\begin{aligned}
X_{c}(r) & =r-z^{\prime}\left[1+\left(z^{\prime}\right)^{2}\right] / z^{\prime \prime}=\rho_{0}\left\{x-y^{\prime}\left[1+\left(y^{\prime}\right)^{2}\right] / y^{\prime \prime}\right\} \\
& =-\rho_{0} \alpha x^{2} \\
Y_{c}(r) & =z(r)+\left[1+\left(z^{\prime}\right)^{2}\right] / z^{\prime \prime}=\rho_{0}\left\{y+\left[1+\left(y^{\prime}\right)^{2}\right] / y^{\prime \prime}\right\} \\
& =\rho_{0}\left[y+(1+\alpha x) \sqrt{(1+\alpha x)^{2}-x^{2}}\right] .
\end{aligned}
$$

In Eq. (10) we use $X_{c}, Y_{c}$ to indicate the center of curvature of the $z(r)$ curve for this purpose treated as if it were in Cartesian coordinates. In particular, we see that $X_{c}$ is negative for $\alpha>0$. Obviously the midgap curve itself is in the $(R, Z)$ plane, and negative values for $R$ are meaningless. It is easily verified from Eq. (10) that $\left(r-X_{c}\right)^{2}+$ $\left(z-Y_{c}\right)^{2}=\rho^{2}(r)$, as it should be.

It is interesting to notice that it is possible to construct a coordinate system with variable center, at $X_{c}, Y_{c}$ and polar coordinates $\eta, \phi$, where $\eta$ is the distance from $X_{c}, Y_{c}$ along the direction of the radius of curvature, and $\phi$ is the angle. Figure 1 depicts this coordinate system which is useful to obtain the anode and cathode curves, once the midgap curve $y(x)$ has been solved for:

$$
\begin{gathered}
\rho_{e}(\phi)=[\rho(\phi) \pm w(\phi) / 2], \quad \phi=\arcsin [x /(1+\alpha x)] \\
\rho(\phi)=\rho_{0}(1+\alpha x)^{2}, \quad w(\phi)=w_{0}(1+\alpha x) .
\end{gathered}
$$

In Eq. (11) $\rho_{e}, \phi$ stands for the anode and cathode coordinates. We obtain the actual $r_{e}, z_{e}$ electrode points using the $\eta, \phi$ center of coordinates defined in Eq. (10),

$$
r_{e}=X_{c}+\rho_{e} \sin \phi, \quad z_{e}=Y_{c}+\rho_{e} \cos \phi .
$$

Any point $R, Z$ inside the TL domain can be thus expressed as

$$
\begin{gathered}
R=X_{c}+\eta \sin \phi, \quad Z=Y_{c}+\eta \cos \phi, \\
\rho-w / 2 \leq \eta \leq \rho+w / 2,
\end{gathered}
$$

where $\rho, X_{c}, Y_{c}$ are given in Eqs. (9) and (10), and $\eta$ is the distance from the polar coordinate center $X_{c}, Y_{c}$ to the $R, Z$ point. 
We can use this coordinate system to obtain $f_{\alpha}=$ $\left(2 \pi / \mu_{0}\right) L$ in terms of the single dimensionless parameter $\alpha$ and elucidate the small gap approximation introduced in Sec. I. Using $\eta, \phi$ coordinates in Eq. (1) yields

$$
\begin{aligned}
f_{\alpha} & =\iint_{A} d R d Z / R \\
& =\int_{\phi_{1}}^{\phi_{2}} d \phi \int_{\rho-w / 2}^{\rho+w / 2} \eta d \eta /\left(X_{c}+\eta \sin \phi\right),
\end{aligned}
$$

where $\phi_{i}=\arcsin \left\{\left[r_{i}-X_{c}\left(r_{i}\right)\right] / \rho\left(r_{i}\right)\right\}, i=1,2$. We can explicitly integrate over $\eta$ obtaining

$$
f_{\alpha}=\int_{\phi_{1}}^{\phi_{2}}\left[w / \sin \phi-X_{c} \ln \left(r_{a} / r_{c}\right) / \sin ^{2} \phi\right] d \phi .
$$

We now expand the logarithm around the midpoint $r$, retaining the first term which is a good approximation if $w \sin \phi / 2 r \ll 1$ :

$$
\begin{aligned}
\ln \frac{r_{a}}{r_{c}} & =\ln \frac{X_{c}+(\rho+w / 2) \sin \phi}{X_{c}+(\rho-w / 2) \sin \phi} \cong w \sin \phi /\left[X_{c}+(\rho \sin \phi)\right] \\
& =w \sin \phi / r, \quad \text { valid for } w \sin \phi / 2 r \ll 1 .
\end{aligned}
$$

Substituting the result of Eq. (16) into Eq. (15) we obtain

$$
\begin{aligned}
f_{\alpha} & =\int_{\phi_{1}}^{\phi_{2}} w / \sin \phi\left(1-X_{c} / r\right) d \phi=\int_{\phi_{1}}^{\phi_{2}} d \phi w \rho / r \\
& =\int_{r_{1}}^{r_{2}} d s(w / r) .
\end{aligned}
$$

The last equality of Eq. (17) gives us back the result anticipated in the Introduction, valid as shown here for $w \sin \phi / 2 r \ll 1$.

As an a posteriori justification of the small gap approximation, we remark that in the example we give below in Fig. 5, having the largest value of $w / 2 r$ of all of the examples we present in this paper, $w_{1} / 2 r_{1}=0.2$ and $w_{2} / 2 r_{2}=0.3$, we find a difference of less than $2 \%$ between that approximation and the actual inductance of the TL of that figure.

It is straightforward to see that Eq. (17) is valid in general given the small gap approximation, without invoking the explicit linear gap dependence. For the particular case we are discussing in this section, where the gap is linear in radius, we replace $w, \rho$, and $r$ using Eqs. (9)-(11) to obtain an expression for the inductance of the optimal TL corresponding to the parameter $\alpha$ as

$$
L_{\alpha}=2 w_{0} \int_{\phi_{1}}^{\phi_{2}} \frac{d \phi}{\sin \phi(1-\alpha \sin \phi)^{2}} .
$$

Before showing the solution to Eq. (7), which has various forms depending on the value of $\alpha$, we remark that it is a first order differential equation which we can integrate analytically for any given $\alpha$. We can then easily impose the condition $y\left(x_{1}\right)=0$. Finally, to have the solution curve going through the outer end point, i.e.z $\left(r_{2}\right)=l_{z}=$ $\rho_{0} y\left(x_{2}\right)$, we vary $\alpha$ using a shooting method to find a value such that $y(x,\{\alpha\})$ satisfies this outer point boundary condition, thus completing the solution.

The various forms for $y(x,\{\alpha\})$ depend on the values of the end points and the AK gaps there. We impose the obvious condition that the argument of the square root in Eq. (7) must be positive over the domain of the radius of the optimal curve.

To aid in the visualization of these different cases as they are being described below, we illustrate in Figs. 3(a)-3(e) the anode, cathode, and midgap curve representative of each case. In all the examples we fix the inner gap $w_{1}=$ $0.6 \mathrm{~cm}$ and the midgap end points to $r_{1}, z_{1}=1.5,0 \mathrm{~cm}$, $r_{2}, z_{2}=6,8 \mathrm{~cm}$. We then vary the outer gap to yield the various cases described below. Also in Fig. 4 we compare the optimal TL with the same end points of gap going linearly from $0.6-0.8 \mathrm{~cm}$ with that of $0.8 \mathrm{~cm}$ constant gap [Fig. 4(a)] and with the midgap straight conical line with the same inner and outer gap [Fig. 4(b)]. The various forms for the solution are as follows.

(i) Circumferential solution, Fig. 3(a).-We recover the Ref. [4] solution by making $\alpha=0$, which represents the constant gap case. From Eq. (9) it is readily seen that the radius of curvature is constant, $\rho(r)=\rho_{0}, \forall r$ being the radius of the circumference described by $z(r)$ as has been presented in Sec. I, and as explained there it can be a double valued with $y^{\prime} \rightarrow \pm \infty$. For this constant gap case we can express the inductance using Eq. (18) setting $\alpha=$ 0 , thus the vacuum inductance in $\mathrm{nH}$ is, with all lengths in $\mathrm{cm}$,

$$
\begin{aligned}
L[\mathrm{nH}] & =2 \int_{\phi_{1}}^{\phi_{2}} d \phi / \sin \phi=2 w \ln \left[\tan \left(\phi_{2} / 2\right) / \tan \left(\phi_{1} / 2\right)\right] \\
\phi_{1} & =\arcsin \left(r_{1} / \rho\right), \\
\phi_{2} & =\arcsin \left(r_{2} / \rho\right) \quad \text { if } l_{z} \leq \sqrt{r_{2}^{2}-r_{1}^{2}} \\
\phi_{2} & =\pi / 2+\arcsin \left(r_{2} / \rho\right), \text { otherwise. }
\end{aligned}
$$

We recall that for this case as explained in Sec. I both $w$ and $\rho$ are constants.

(ii) Circumferential-like case $r_{2} k / w_{2}<\alpha<1$, Fig. 3(b).-The analytical solution of Eq. (7) is given by

$$
\begin{aligned}
y= & f(x)-f\left(x_{1}\right) \\
f(x)= & \frac{\alpha \arcsin \left[\left(1-\alpha^{2}\right) x-\alpha\right]}{\left(1-\alpha^{2}\right)^{3 / 2}} \\
& -\frac{\sqrt{-\left(1-\alpha^{2}\right) x^{2}+2 \alpha x+1}}{\left(1-\alpha^{2}\right)} .
\end{aligned}
$$

In Eq. (20) $y\left(x_{1}\right)=0$ manifestly, thus satisfying one of the two boundary conditions. We have called this case "circumferential-like" because a real solution only exists up to a maximum value of $x=x_{\max }=1 /(1-\alpha)$ for which $y^{\prime} \rightarrow \pm \infty, x \rightarrow x_{\max }$. Thus, if for a given value of 

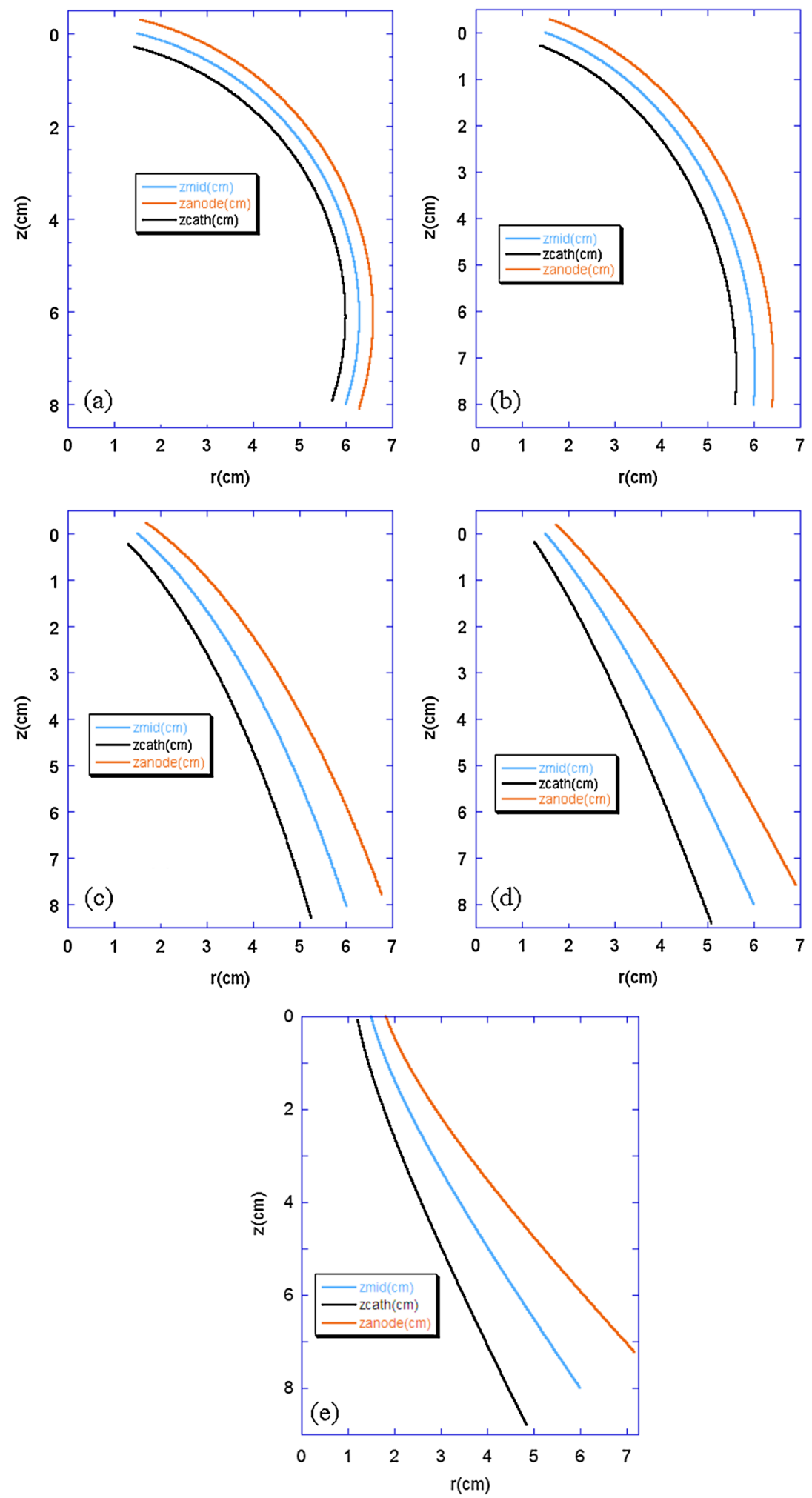

FIG. 3. (Color) Electrode and midgap curves for optimal TL (cathode in black, anode in red, midgap in blue) of gaps varying linearly with radius. They all have the same inner end point and outer end points at $r_{1}=1.5 \mathrm{~cm}, z_{1}=0 \mathrm{~cm}, r_{2}=6 \mathrm{~cm}, z_{2}=l_{z}=8 \mathrm{~cm}$. In all cases the inner point gap is $0.6 \mathrm{~cm}$. The outer midgap end point gap is varied: (a) solution in Ref. [4], $0.6 \mathrm{~cm}$, (b) $0.8 \mathrm{~cm}$, (c) $1.6 \mathrm{~cm}$, (d) $2.0 \mathrm{~cm}$, and (e) $2.8 \mathrm{~cm}$. Notice the change of character of the optimal TL as the outer gap increases, from doubled-value midgap curve to straight-biconic-like, to changing from concave to convex in (e), as explained in the text. The vacuum inductances of the optimal TL in each of the plots compared to the straight-line TL are in $\mathrm{nH}$ as follows: $\left[L_{\text {optimal }}, L_{\text {straight }}\right] \mathrm{nH}$ : (a) [2.9, 3.4], (b) [3.5, 3.9], (c) $[5.5,5.6]$, (d) $[6.4,6.5]$, and (e) $[8.2,8.2]$. 

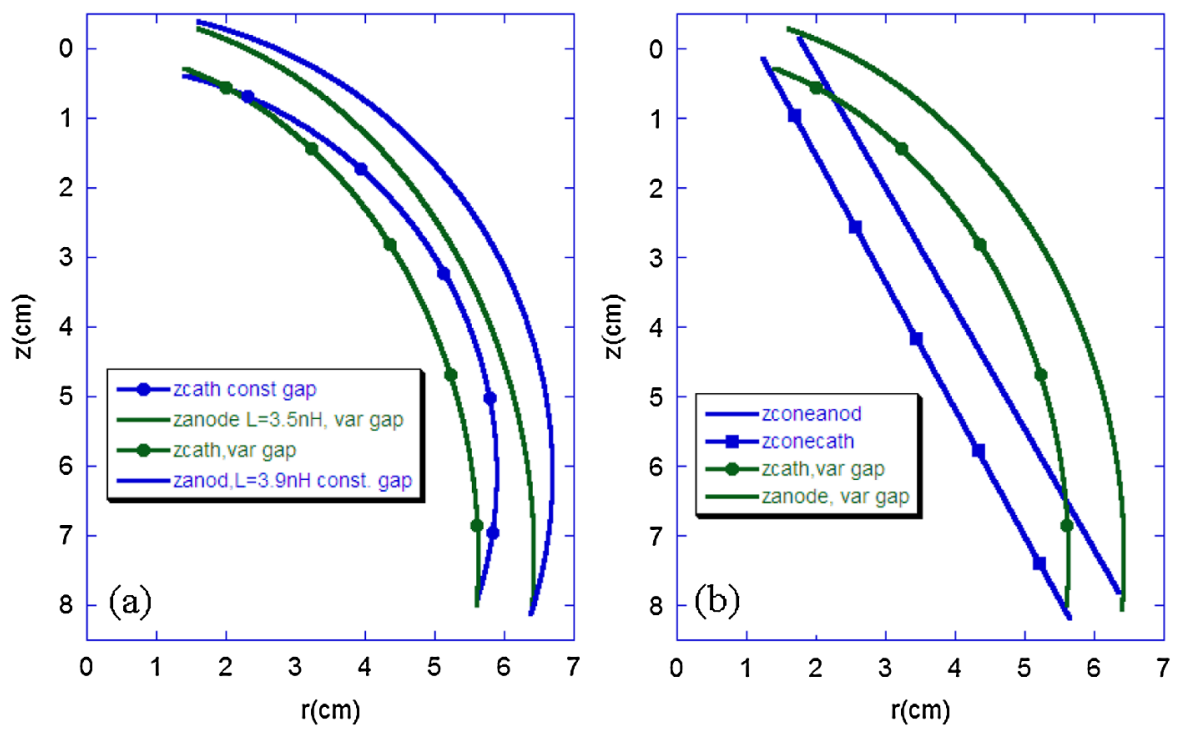

FIG. 4. (Color) These two figures, (a) and (b), show the electrode curves (cathodes marked with circles) for the optimal TL shown in Fig. (b). In (a) the comparison is between the optimal line of $0.8 \mathrm{~cm}$ constant gap and varying the gap as explained in the captions of Fig. 3 linearly from 0.6 to $0.8 \mathrm{~cm}$, resulting in decreasing the vacuum inductance from 3.9 to $3.5 \mathrm{nH}$ as it was described in the caption of Fig. 3. The optimal line of constant gap is in green. In (b) the comparison is between the optimal TL of Fig. 3(b) in green, and a straight-line midgap TL, in blue, with the same end points and end gaps. Vacuum inductances are [3.5, 3.8] $\mathrm{nH}$, respectively.

$\alpha$ within the range being considered in this case $y\left(x_{\max }\right)<$ $l_{z} / \rho_{0}$, for the second boundary condition to be satisfied it follows that $y(x)$ must be double valued, just as when $\alpha=$ 0 . Thus, for the second branch for this case we have

$$
\bar{y}(x)=2 y\left(x_{\max }\right)-y(x), \quad x_{\max } \geq x \geq x_{2} .
$$

In Eq. (21) $\bar{y}(x)$ denotes the second branch values of the solution curve in terms of the first branch $y(x)$ given in Eq. (20).

(iii) Straight-line cone (SLC)-like solution, Figs. 3(c) and 3(d), $\alpha>1$.- The analytical solution of Eq. (7) is given by

$$
\begin{aligned}
y= & f(x)-f\left(x_{1}\right) \\
f(x)= & \frac{q(x)}{\left(\alpha^{2}-1\right)} \\
& -\frac{\alpha \ln \left\{2\left[\left(\alpha^{2}-1\right) x+\alpha+\left(\alpha^{2}-1\right)^{1 / 2} q(x)\right]\right\}}{\left(\alpha^{2}-1\right)^{3 / 2}} \\
q(x)= & \sqrt{\left(\alpha^{2}-1\right) x^{2}+2 \alpha x+1} .
\end{aligned}
$$

The function $y(x)$ is as before constructed to satisfy the boundary condition $y\left(x_{1}\right)=0$. From Eq. (22) it is clear that when $\alpha \geq 1$ the argument of the square root given by $q(x)$ is always positive implying that $y^{\prime}$ stays finite and positive. In that sense this solution shares the character of the SLC case.

(iv) Straight-line cone ( $S L C$ ) solution.-A special case to notice is given by $w_{0}=0$, formally corresponding to $\alpha$, $\rho_{0} \rightarrow \infty$. In this case Eq. (6) becomes

$$
z^{\prime} / \sqrt{1+\left(z^{\prime}\right)^{2}}=C / k,
$$

whose solution is constant $z^{\prime}$ : the midgap curve is a straight line in the $R, Z$ plane going through the end points:

$$
z(r)=l_{z}\left(r-r_{1}\right) /\left(r_{2}-r_{1}\right)
$$

For this case it is straightforward to obtain the inductance from Eq. (1) recalling $w=k r$ and from Eq. (24) $z^{\prime}=$ $l_{z} /\left(r_{2}-r_{1}\right)$, thus the vacuum inductance in $\mathrm{nH}$ is, with all lengths in $\mathrm{cm}$,

$$
\begin{aligned}
L[\mathrm{nH}] & =2 k\left(r_{2}-r_{1}\right) \sqrt{1+l_{z}^{2} /\left(r_{2}-r_{1}\right)^{2}} \\
& =2\left(w_{2}-w_{1}\right) \sqrt{1+l_{z}^{2} /\left(r_{2}-r_{1}\right)^{2}} .
\end{aligned}
$$

(v) Limited solution case, $w_{0}<0,-\alpha>k r_{1} / w_{1}>0$, Fig. 3(e).-This case is very much like the one in (iii), but we show below that in some instances no optimal solution exists. The solution is given by

$$
\begin{aligned}
y= & f(x)-f\left(x_{1}\right) \\
f(x)= & \frac{q(x)}{\left(\alpha^{2}-1\right)} \\
& -\frac{\alpha \ln \left\{2\left[\left(\alpha^{2}-1\right) x+\alpha+\left(\alpha^{2}-1\right)^{1 / 2} q(x)\right]\right\}}{\left(\alpha^{2}-1\right)^{3 / 2}} \\
q(x)= & \sqrt{\left(\alpha^{2}-1\right) x^{2}+2 \alpha x+1} .
\end{aligned}
$$

From Eq. (26), keeping in mind that $\alpha<0$ it follows that $y^{\prime} \rightarrow \pm \infty, x \rightarrow x_{\min }=1 /(1-\alpha)$. This implies that values of $x$ such as $x_{\min } \leq x \leq x_{1}$ are possible and the solution 
could be double valued. This is clearly against our imposed constraint that the optimal curve does not go to radii smaller than the inner one. It also follows from analyzing the roots of the argument of the square root in $q(x)$ that only values of $\alpha<-1$ are acceptable so that the argument of the square root is positive in the interval $x_{1} \leq x \leq x_{2}$. Thus the values of $\alpha$ that guarantee that the argument of the square root is strictly positive and that $x_{1} \geq x_{\min }=1 /(1-$ $\alpha)$ are

$$
\alpha \leq \alpha_{\max }=-k r_{1} / w_{1},
$$

where $k r_{1} / w_{1}=\left(w_{1}-w_{0}\right) / w_{1}>1$ for $w_{0}<0$.

Moreover, for this limiting value of $\alpha_{\max }$ the length along the $z$ axis $z\left(r_{2}\right)-z\left(r_{1}\right)$ is the maximum attainable. This can be seen integrating Eq. (7) and going back to $r, z$ variables:

$$
\Delta z=z\left(r_{2}\right)-z\left(r_{1}\right)=\int_{r_{1}}^{r_{2}} r / \sqrt{\rho_{0}^{2}\left(1+r k / w_{0}\right)^{2}-r^{2}} .
$$

The denominator of the integrand of Eq. (28) is positive and increases for increasing values of $\rho_{0}$ for all values of $r_{2} \geq r \geq r_{1}$, because

$$
\begin{gathered}
\left.\frac{\partial \rho_{0}^{2}\left(1+r k / w_{0}\right)^{2}-r^{2}}{\partial \rho_{0}}\right|_{r}=2 \rho_{0}\left(w_{1} / w_{0}\right)^{2}>0, \\
\forall r_{2} \geq r \geq r_{1} .
\end{gathered}
$$

This means that the lowest allowed value of $\rho_{0}$-highest allowed $\alpha$-corresponds to the highest attainable value of $\Delta z$. Therefore if the condition

$$
\left.\Delta z\right|_{\max }=z\left(r_{2},\left\{\alpha_{\max }\right\}\right)-z\left(r_{1},\left\{\alpha_{\max }\right\}\right)<l_{z}
$$

applies, then there is no optimal solution satisfying the boundary conditions and our constraint $x_{1} \geq x_{\min }$. The limiting curve has the property that $y^{\prime}\left(x_{\min }=x_{1}\right)=\infty$. We remark that the solution for $w_{0}<0$, given by Eq. (26), is convex. In all other cases with $w_{0} \geq 0$ the solution is concave. This can be seen by noticing that $X_{c}>$ 0 for $\alpha<0$ from Eq. (10). The characteristic behavior of the solution curves for $w_{0}<0$ as they approach the limiting curve, the one reaching the maximum attainable TL axial length, is depicted in Fig. 5.

To obtain the inductance corresponding to optimal curves we rewrite Eq. (1) as

$$
L=\frac{\mu_{0} w_{0}}{2 \pi} \int_{x_{1}}^{x_{2}}(1+\alpha x) \sqrt{1+\left(y^{\prime}\right)^{2}} d x / x .
$$

From Eq. (7) we have that the integrand in Eq. (31) can be written as

$$
\sqrt{1+\left(y^{\prime}\right)^{2}}(1+\alpha x) / x=\left[1+\left(y^{\prime}\right)^{2}\right] / y^{\prime} .
$$

By substituting the integrand in Eq. (31) by the expression in Eq. (32) we find

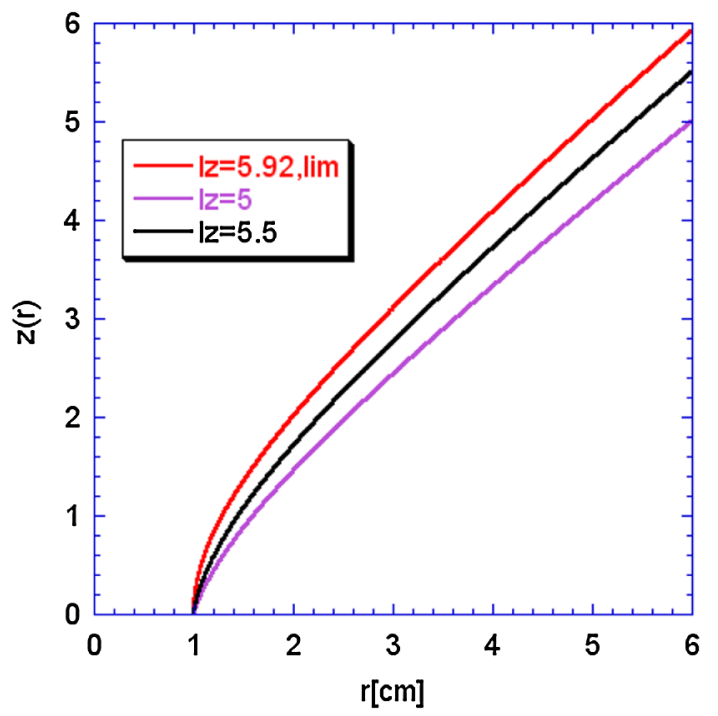

FIG. 5. (Color) Optimal $z(r)$ for our solution for case (v) in Sec. II, i.e. $w_{0}<0$. It shows the midgap curves for three optimal $\mathrm{TL}$, all of them having the same inner point and gap $r_{1}=1 \mathrm{~cm}$, $z_{1}=0 \mathrm{~cm}$, and $w_{1}=0.4 \mathrm{~cm}$. The outer end point radius and gap are also fixed at $r_{2}=6 \mathrm{~cm}, w_{2}=3.6 \mathrm{~cm}$, corresponding to $w_{0}=-0.24 \mathrm{~cm}$ The outer point axial position is varied from $z_{2}=5 \mathrm{~cm}$ to $z_{\text {lim }}=5.92 \mathrm{~cm}$. This latter value for the axial position is such that $z^{\prime} \rightarrow \infty, r=r_{1}$. A higher value of the axial position is not attainable without going to radii smaller than $r_{1}$, but this would violate the constraint of not allowing any curve going through radii smaller than that of the inner point.

$$
\begin{aligned}
L & =\frac{\mu_{0} w_{0}}{2 \pi} \int_{x_{1}}^{x_{2}} d x\left(y^{\prime}+1 / y^{\prime}\right) \\
& =\frac{\mu_{0} w_{0}}{2 \pi \rho_{0}}\left(l_{z}+\int_{0}^{l z / \rho_{0}}(d x / d y)^{2} d y\right) .
\end{aligned}
$$

In Eq. (33) we use the inverse function $x(y)$ because it is single valued and its derivative is always finite. The integral in Eq. (33) is calculated numerically by a straightforward 1D quadrature once we have solved for the optimal function $y(x)$. The inductance of the optimal TL can thus be calculated using Eq. (33) or Eq. (18), comparing both gives a test of numerical accuracy of the procedure. In all cases we find that the two methods result in inductances differing by less than $0.1 \%$.

\section{NUMERICAL SOLUTION FOR AK GAP HAVING NONLINEAR DEPENDENCE ON RADIUS}

For AK gaps which depend only on radius but not linearly, we sketch a method of numerical solution. Equation (6) is applicable, because we have only assumed $w=w(r)$ to obtain it. From it we have

$$
z(r)-z\left(r_{1}\right)=\int_{r_{1}}^{r} d r C r / \sqrt{w^{2}-C^{2} r^{2}} .
$$

Thus for any given value of the dimensionless constant $C$, Eq. (34) is a simple 1D integral straightforward to perform 
as long as

$$
C<w / r, \quad r_{2} \geq r \geq r_{1} .
$$

The solution is then obtained by finding $C$ such that the integral of Eq. (34) satisfies $z\left(r_{2}\right)-z\left(r_{1}\right)=l_{z}$.

The method becomes particularly transparent if we impose that $w / r$ be a monotonically decreasing function of radius, that is

$$
d(w / r) / d r<0 .
$$

When the condition of Eq. (36) is satisfied it follows from Eq. (34) that the solution can become double valued, as was described for the appropriate cases in the previous section. The relationship between $C$ and $\bar{r}$, the maximum radius for which $z^{\prime} \rightarrow \pm \infty$ is

$$
C=w(\bar{r}) / \bar{r} .
$$

As in the linear case, depending on the boundary conditions, the solution is either SLC-like or circumferentiallike.

The radius of curvature is given by

$$
\rho=\frac{w^{2} / C}{\left(w-r w^{\prime}\right)}
$$

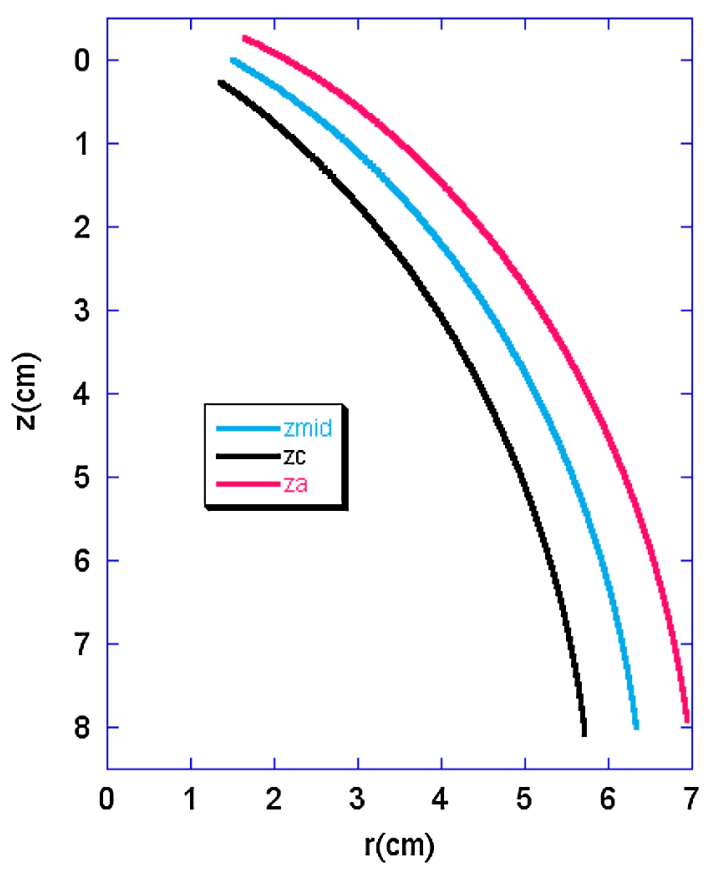

FIG. 6. (Color) Optimal TL, gap varies as $r^{1 / 2}$ from 0.6 to $1.2 \mathrm{~cm}$. Electrode and midgap curves (cathode in black, anode in red, midgap in blue) for a TL whose gap varies as the square root of radius, being $w_{1}=0.6 \mathrm{~cm}$ at the inner end point, $r_{1}=$ $1.5 \mathrm{~cm}, z_{1}=0 \mathrm{~cm}$, and $w_{2}=1.2 \mathrm{~cm}$ at the outer end point $r_{2}=$ $6 \mathrm{~cm}, z_{2}=l_{z}=8 \mathrm{~cm}$. As explained in the text this curve was obtained by numerical integration of Eq. (34), varying the $\rho_{1}$ defined in Eq. (39) until the resulting optimal $z(r)$ goes through the indicated end points. The vacuum inductance of this optimal $\mathrm{TL}$ is $4.4 \mathrm{nH}$.
For functions $w(r)$ such that $w_{0}=w(0)>0$ and $r w^{\prime} \rightarrow 0$ at the origin, it is straightforward to see that $\rho_{0}=\rho(0)=$ $w_{0} / C$ as it was for the linear case.

As an example we show in Fig. 6 the optimal midgap and electrode curves for a TL for which $w=w_{1}\left(r / r_{1}\right)^{1 / 2}$ obtained as described in this section. For ease of comparison we used the same end points as in Fig. 3 and the same inner point gap of $0.6 \mathrm{~cm}$, thus resulting in an outer gap of $w_{2}=$ $0.6 \mathrm{~cm}(6 / 1.5)^{1 / 2}=1.2 \mathrm{~cm}$. We obtained $L=4.4 \mathrm{nH}$ for the vacuum inductance for this TL, which is intermediate between the linear case examples given in Sec. II for outer gaps 0.8 and $1.6 \mathrm{~cm}, L=3.5,5.5 \mathrm{nH}$ respectively [cases (ii) and (iii)].

For this functional form for the gap as a function of radius it follows that the midgap curve radius of curvature is given by

$$
\rho=\rho_{1}\left(r / r_{1}\right)^{1 / 2}, \quad \rho_{1}=\rho\left(r_{1}\right)=2 w_{1} / C .
$$

\section{CONCLUSIONS}

We have extended Ref. [4] inductance minimization for axisymmetric TL of constant gap to those for which the AK gap is allowed to be a function of radius. For linear dependence we presented the analytical form for the minimum inductance TL for various cases as a function of a single dimensionless parameter. This parameter in turn is found numerically by imposing that the midgap curve go through prescribed end points in the $R, Z$ plane. We also introduced a simple method of solution for TL having AK gap with nonlinear radial dependence. We showed how to calculate inductance by performing numerically a 1D quadrature, once the optimal TL has been obtained.

The largest savings in inductance between the optimal TL and the nonoptimal TL with straight-line midgap curve, going through the same end points and same end point gaps varying linearly with radius, is realized when the axial extent of the line is large compared to its radial extent. Thus, in the example shown in Fig. 2-constant gap-we see an inductance saving of $36 \%$ from a coaxial line to the optimal one (circumferential) for end points at the same radius, $r_{1}=r_{2}$. We remark that for linear gap dependence on the radius, the only TL with straight-line midgap that is optimal is the one we have described in case (iv) of Sec. II as SLC, for which the gap extrapolated to the axis is $w_{0}=0$.

We see about $10 \%$ savings going between the optimal lines in the example shown in Fig. 4(a) when allowing the gap to vary as indicated there. Finally, we show about 5\% lower inductance in the example given in Fig. 4(b) when comparing the optimal line with the straight-line one with same initial and final gaps. This small difference is explained by the fact that the TL of Fig. 4(b) has comparable line axial and radial extent. This observation of modest inductance savings between optimal and straight-line TL is also confirmed in all the examples given in Fig. 3 in which 
the initial and final gaps are such that the solution is not circumferential-like. The inductance comparison for all examples given can be found in their figure captions.

Two important aspects need to be remarked. The first is that-as in Ref. [4] —our present solution imposes as boundary conditions that the midgap curve go through the prescribed end points. Thus, once the solution for that curve is obtained the electrode surfaces are given uniquely. Therefore, if the required connections between the pulsedpower driver and the load do not exactly match the end points of optimal TL electrode surfaces given by this solution, extra hardware needs to be designed. For example, overall smoothness and curvature constraints are usually important in the design of such connections. The inductance of these connections also needs to be evaluated and added to that of the optimal TL solution.

The second aspect is that the actual dependence of the AK gap on radius follows from criteria outside of the scope of this work. Clearly here we have chosen a static criterion, not including any dynamic behavior, such as, for instance, the time-dependent closure of the AK gaps by electrode plasmas, or the time dependence of the electric to the magnetic field ratio describing magnetic insulation and electron flow currents. The minimization of flow current, not investigated in this work, is a common design criterion for multi-mega-ampere, multimodule pulsed-power drivers $[1,2,5]$. The technique presented in this paper should prove useful in assessing a given TL even when it is not employed in its actual design, or in providing a starting point for simulations that include additional physics such as electron flow or electrode plasmas. This solution provides the lowest attainable inductance given the AK gap radial dependence and the midgap curve prescribed end points. Thus, even if connections to the input and output of the optimal solution or other considerations prevent the use of this technique, it still provides a figure of merit to assess how close the actual TL design inductance is from the absolute attainable minimum.

\section{ACKNOWLEDGMENTS}

We acknowledge helpful conversations with Dr. O.A. Hurricane, Dr. R.W. Lemke, Dr. C. A. Jennings, and Dr. W. A. Stygar. Sandia is a multiprogram laboratory operated by Sandia Corporation, a Lockheed Martin Company, for the United States Department of Energy's National Nuclear Security Administration under Contract No. DE-AC04-94AL85000.

[1] W. A. Stygar, R. B. Spielman, G. O. Allshouse, C. Deeney, D. R. Humphreys, H. C. Ives, F.W. Long, T. H. Martin, M. K. Matzen, D. H. McDaniel, C. W. Mendel, Jr., L.P. Mix, T. J. Nash, J.W. Poukey, J. J. Ramirez, T. W.L. Sanford, J.F. Seamen, D. B. Seidel, J.W. Smith, D. M. Van De Valde, R.W. Wavrik, P. A. Corcoran, J.W. Douglas, I.D. Smith, M. A. Mostrom, K. W. Struve, T.P. Hughes, R.E. Clark, R.W. Shoup, T.C. Wagoner, T.L. Gilliland, and B. Peyton, in Proceedings of the 11th IEEE International Pulsed Power Conference, edited by G. Cooperstein and I. Vitkovitsky (IEEE, Piscataway, NJ, 1997), p. 591.

[2] W. A. Stygar et al., Phys. Rev. ST Accel. Beams 9, 090401 (2006).

[3] W. A. Stygar et al., Phys. Rev. E 69, 046403 (2004).

[4] O. A. Hurricane, J. Appl. Phys. 95, 4503 (2004).

[5] J.P. Martin, M.E. Savage, T.D. Pointon, and M. A. Gilmore, Phys. Rev. ST Accel. Beams 12, 030401 (2009).

[6] Richard Stevens Burington, Handbook of Mathematical Tables and Formulas (McGraw-Hill Book Company, New York, 1965), 4th ed., p. 52. 